\title{
CLASSIFICATION OF STABLE CURRENTS IN THE PRODUCT OF SPHERES
}

\author{
SHIHSHU WALTER WEI
}

In [11] we constructed the first set of examples of area-minimizing hypersurfaces with isolated singularities in various high dimensional locally symmetric spaces of both compact type and non-compact type. These are not the minimizing cones in Euclidean spaces $\mathbb{R}^{n}$ constructed by Bombieri, de Giorgi and Giusti [2] and later by Lawson [6].

Among our first known examples in various locally symmetric spaces, the ones in the product of hyperbolic spaces $H^{n+1} \times H^{n+1}$ are complete, while the examples in the product of Euclidean spheres $S^{n+1} \times S^{n+1}$ have boundaries, where $n>2$. From the analytic viewpoint, this is due to the fact that the non-linear elliptic partial differential inequalities involved in $S^{n+1} \times S^{n+1}$ unlike the ones in the non-compact type case, have only local solutions.

This striking contrast leads immediately to the conjecture that no closed stable rectifiable $(2 n+1)$-current (or roughly speaking, no closed stable minimal hypersurface with singularities) exists in $S^{n+1} \times S^{n+1}$, and hence motivates a general study in $S^{p} \times S^{q}$. A stable rectifiable current (resp. stable minimal submanifold) is a stationary or minimal rectifiable current (resp. minimal submanifold) that has no mass (resp. area) decreasing variations. An extension of Synge lemma due to Simons [9] which states that there are no closed stable hypersurfaces in a Riemannian manifold with positive Ricci curvature supports a piece of evidence of the conjecture.

In this paper, we give a positive answer to the conjecture and classify the stable currents in the product of Euclidean spheres $S^{p} \times S^{q}$.

Theorem. (Classification Theorem) For $p$ and $q \neq 1$ or 2, the only closed stable rectifiable currents in the product of Euclidean spheres $S^{p} \times S^{q}$ are $S^{p} \times\{p t$. $\}$ or $\{p t.\} \times S^{q}$ or sums of these. In fact, any closed varifold of dimension $\neq p$ or $q$ is not stable, and neither is the diagonal embedding of $S^{n}$ into $S^{n} \times S^{n}$ for $n>2$. In $S^{2} \times S^{2}$, the only closed stable rectifiable currents are holomorphic or anti-holomorphic or sums of these. Hence, the diagonal and skew-diagonal embeddings of $S^{2}$ into $S^{2} \times S^{2}$ are both stable.

The technique is to first prove a decomposition theorem for the tangent space to the support of a current in the product of Riemannian manifolds (cf. Theorem 1). Then derive a trace 
formula (Proposition 2), and make sharp estimates, case by case, of the trace of a quadratic form involving the second variational formula over a vector space of certain selected "distinguished" vector fields in the product of spheres along which the deformation of a current is being performed. Along with many other results, these trace estimates imply that a current $T$ in $S^{p} \times S^{q}$ ( $p$ and $q \neq 1$ or 2) is stable if and only if $T$ is either of dimension $p$ or $q$ with tangent vectors almost everywhere lying in one factor of the tangent space to $S^{p} \times S^{q}$. We conclude that these stable currents are in fact totally geodesic submanifolds $S^{p}$ or $S^{q}$ by a beautiful characterization technique [8] in geometric measure theory.

It should be pointed out that the average process from the calculus of variations was first employed by Lawson and Simons [7]. They have shown that there are no closed stable varifolds in the Euclidean sphere $S^{n}$, and the only closed stable rectifiable currents in the complex projective space $C P^{n}$ are algebraic cycles.

While the work generalizes an idea of Synge [10], study global geometry and topology by deforming a geodesic along its parallel normal vector field, one can deform any current along a "universally" fixed collection of distinguished vector fields to solve global problems by averaging.

The method to classify the stable currents in $S^{p} \times S^{q}$ can be carried over to a more general setting. Namely, it can be applied to the study of topology of submanifolds in $S^{n} \times S^{m}$ as well as in Yang-Mills fields. We have discussed these phenomena in another paper [12].

The method can be extended to an extrinsic average variational method ([15]), for various manifolds that are not symmetric spaces, and for various functionals that are not area and mass functionals (for the motivation, see also [16, p.626]). The extended method ([15]) was applied to harmonic maps by R. Howard and S.W. Wei, $p$-harmonic maps by S.W. Wei and C.M. Yau, and F-harmonic maps by M. Ara to introduce and study the notions of superstrongly unstable manifold, $p$-superstrongly unstable manifold, and $F$-superstrongly unstable manifold ([5],[18],[1]). The extended method has also been applied to establish some topological vanishing theorems for higher homotopy groups ([16],[17]). This is in contrast to [7] and [12], where topological vanishing theorems for homology groups are studied.

The author wishes to thank Professors H. Blaine Lawson Jr., and Ralph Howard for their helpful discussions, and Professor Herman R. Gluck for his interest. The author also wishes to express his gratitude to the editor, the staff, and Tamkang Journal of Mathematics for making the present form of the paper possible.

\section{Definitions and preliminary remarks}

To solve the variational problems (e.g. oriented Plateau's problem) and represent the homology groups of a manifold $N$, Federer and Fleming [4] introduced rectifiable currents 
which may be thought of as an oriented surface with singularities. They have tangent planes almost everywhere and can be strongly approximated by smooth surfaces.

For the purpose of completeness and calculation, we shall adopt the notation developed in detail in [7] and outline briefly below. For convenience $N$ can be considered as embedded in some Euclidean space. We begin with the Hausdorff $p$-measure $\mathscr{H}^{p}$ of a set $S \subset N$ defined by

$$
\mathscr{H}^{p}(S)=\lim _{\epsilon \rightarrow 0^{+}} \inf \left\{\sum_{\theta \in C} \alpha_{p}\left(\frac{\operatorname{diam} \theta}{2}\right)^{p}, C \in \mathscr{C}_{\epsilon}(S)\right\}
$$

where $\mathscr{C}_{\epsilon}(S)$ is the collection of countable coverings of $S$ by sets of diameter $\leq \epsilon$ and $\alpha_{p}$ is the $p$-area of the unit $p$-ball. An $\mathscr{H}^{p}$-measurable set $S \subset N$ with $\mathscr{H}^{p}(S)<\infty$ is called $p$ rectifiable if for all $\epsilon>0$, there is an embedded $C^{1}$ submanifold $S^{\prime}$ of dimension $p$ such that $\mathscr{H}^{p}\left(S \Delta S^{\prime}\right)<\epsilon$, where $\Delta$ denotes symmetric difference.

We call $(S, \xi)$ an oriented $p$-rectifiable set if $S$ is a $p$-rectifiable set and $\xi$ an $\mathscr{P}^{p}$-measurable section $\xi: S \rightarrow \wedge^{p} T(N)$ of the $p$-th exterior product of the tangent-bundle of $N$ with the property that for $\mathscr{H}^{p}$ - almost all $x \in S, \xi_{x}$ is a simple vector of unit length which represents $T_{x}(S)$.

Therefore, given an oriented $p$-rectifiable set $\mathscr{S}=(S, \xi)$ and a smooth $p$-form $\omega$, we can interpret

$$
\mathscr{S}(\omega)=\int_{S} \omega\left(\xi_{x}\right) d \mathscr{H}^{p}(x)
$$

as a continuous linear functional on the space $\wedge^{p}(N)$ of differential $p$-forms on $N$ with the sup-norm topology. Furthermore, the norm $\mathbf{M}(\mathscr{S})=\mathscr{H}^{p}(\mathscr{S})$ and the $\mathbf{M}$-closure of the group generated by the oriented $p$-rectifiable sets in the dual space of $\wedge^{p}(N)$ is called the set of rectifiable $p$-currents and shall be denoted by $\mathfrak{R}_{p}(N)$. It is known that for any $\mathscr{S} \in \mathfrak{R}_{p}(N)$

$$
\mathscr{S}=\sum_{n}^{\infty} n \mathscr{S}_{n}
$$

where $\mathscr{S}_{n}$ are disjoint collections of oriented $p$-rectifiable set $\left(S_{n}, \xi_{n}\right)$ with $\mathbf{M}(\mathscr{S})=\sum_{1}^{\infty} n \mathscr{H}^{p}\left(S_{n}\right)$ $<\infty$. Hence we associate with each $\mathscr{S} \in \mathfrak{R}_{p}(N)$, a Borel measure $\|\mathscr{S}\|$ called the total variation measure of $\mathscr{S}$.

For every $x \in S_{n}$, we define $\vec{S}_{x}=\xi_{n}(x)$. Thus $\vec{S}$ denotes the field of oriented tangent planes of $\mathscr{S}$. The boundary of $\mathscr{S}$ is given by

$$
(\partial \mathscr{S})(\omega)=\mathscr{S}(d \omega)
$$

where $d$ denotes exterior differentiation. $\mathscr{S}$ is an integral current if $\mathscr{S}$ and $\partial \mathscr{S}$ are rectifiable. We call $\mathscr{S}$ an $m$-dimensional normal current if $S$ and $\partial \mathscr{S}$ are representable by integration (or $m=0$ ) with compact support. 
Just as rectifiable currents are defined as $Z$-chains of oriented rectifiable sets, so are rectifiable $G$-currents $\Re_{p}(G)$ defined to be $G$-chains of oriented $p$-rectifiable sets

$$
\mathscr{S}=\sum_{g \in G-\{0\}} g \mathscr{S}_{g}
$$

where $\left\{\mathscr{S}_{g}=\left(S_{g}, \xi_{g}\right): g \in G\right\}$ is a disjointed family of oriented $p$-rectifiable sets with

$$
\mathbf{M}(S)=\sum|g| \mathscr{H}^{p}\left(S_{g}\right)<\infty
$$

We can define the support of $G$-current $\mathscr{S}$, the field of unit tangent $p$-planes, and the variation measure $\|\mathscr{S}\|$ in an analogous way.

A $p$-dimensional varifold on $N$ is a radon measure on the total space of the fiber bundle $G_{p}^{\prime}(N) \stackrel{\pi}{\rightarrow} N$ of unoriented $p$-dimensional subspaces of tangent space of $N$.

A current $T \in \mathfrak{R}_{k}(N)$ is said to be stationary or minimal if $\left.\frac{d}{d t} \mathbf{M}\left(\phi_{t^{*}}^{V}(T)\right)\right|_{t=0}=0$ for all vector fields $V$ on $N$ with compact support where $\phi_{t}^{V}$ is the flow associated with $V$. A current $T \in \mathfrak{R}_{k}(N)$ is said to be stable if for every vector field $V$ with compact support, there exists an $\epsilon>0$ such that

$$
\mathbf{M}(T) \leq \mathbf{M}\left(\phi_{t^{*}}^{V}(T)\right)
$$

for $|t|<\epsilon$.

Note that if $T$ is stable, then for each $V$ we have

$$
\begin{gathered}
\left.\frac{d}{d t} \mathbf{M}\left(\phi_{t^{*}}^{V}(T)\right)\right|_{t=0}=0 \\
\left.\frac{d^{2}}{d t^{2}} \mathbf{M}\left(\phi_{t^{*}}^{V}(T)\right)\right|_{t=0} \geq 0 .
\end{gathered}
$$

A current $T \in \mathfrak{R}_{k}(N)$ is homologically (or absolutely) mass-minimizing over $Z$ if for all compact sets $K \subset N$, we have

$$
\mathbf{M}\left(\phi_{K} T\right) \leq \mathbf{M}\left(\left(\phi_{K} T\right)+\mathscr{S}\right)
$$

for all $\mathscr{S} \in \mathfrak{R}_{k}^{l o c}(N)$ have compact support and boundary of a current in $\mathfrak{R}_{k+1}^{l o c}(N)$ (or zero), where $\phi_{K}$ is the characteristic function on $K$.

Suppose now that $N^{n}$ is an $n$-dimensional complex manifold with almost complex structure $J$. A current $\mathscr{S} \in \mathfrak{R}_{2 p}(N)$ is holomorphic if $J \vec{S}_{x}=0$ for $\|\mathscr{S}\|$ almost all $x$. A current $\mathscr{S} \in \mathfrak{R}_{2 p}(N)$ is anti-holomorphic if $J \vec{S}_{x}$ is orthogonal to $\vec{S}_{x}$ for $\|\mathscr{S}\|$ almost all $x$. 


\section{A decomposition theorem in general product spaces}

Theorem 1. Let $x=\left(x_{1}, x_{2}\right)$ be a point in the product manifolds $M_{1} \times M_{2}$ of Riemannian manifolds $M_{1}$ and $M_{2}$. Suppose the support of a rectifiable $p$-current in $\Re_{p}\left(M_{1} \times M_{2}\right)$, or $\Re_{p}\left(M_{1} \times\right.$ $\left.M_{2}, G\right)$, or a $p$-varifold on $M_{1} \times M_{2}$ has a tangent space $\mathbb{W}$ at $\left(x_{1}, x_{2}\right)$. Then there exist $i, j, k \geq 0$ such that $i+j+k=p$, and an orthonormal basis $\mathscr{B}$ for $\mathbb{W}$, of the form $\mathscr{B}=\mathscr{B}_{1} \cup \mathscr{B}_{2} \cup \mathscr{B}_{3}$, where $\mathscr{B}_{1}=\left\{\bar{e}_{1}, \cdots, \bar{e}_{i},\right\} \subset T_{x_{1}}\left(M_{1}\right), \mathscr{B}_{2}=\left\{\overline{\bar{e}}_{1}, \cdots, \overline{\bar{e}}_{j}\right\} \subset T_{x_{2}}\left(M_{2}\right)$, and $\mathscr{B}_{3}=\left\{e_{1}=a_{1}+b_{1}, \cdots, e_{k}=\right.$ $\left.a_{k}+b_{k}\right\}$ with $\left\{a_{1}, \cdots, a_{k}\right\} \subset T_{x_{1}}\left(M_{1}\right)$, and $\left\{b_{1}, \cdots, b_{k}\right\} \subset T_{x_{2}}\left(M_{2}\right)$, such that

$$
\left\langle a_{\ell}, a_{\ell^{\prime}}\right\rangle=\left\langle b_{\ell}, b_{\ell^{\prime}}\right\rangle=\left\langle a_{\ell}, \bar{e}_{s}\right\rangle=\left\langle b_{\ell}, \overline{\bar{e}}_{t}\right\rangle=0,
$$

for every $\ell \neq \ell^{\prime}$, where $1 \leq \ell, \ell^{\prime} \leq k, 1 \leq s \leq i$, and $1 \leq t \leq j$. If $i=0$ (resp. $j=0, k=0$ ), we define $\mathscr{B}_{1}=\varnothing,\left(\right.$ resp. $\mathscr{B}_{2}=\varnothing, \mathscr{B}_{3}=\varnothing$.) In particular, both $a_{\ell}$ and $b_{\ell}$ are orthogonal to $\mathbb{W}_{1} \oplus \mathbb{W}_{2}$, where $\mathbb{W}_{\iota}=\mathscr{W} \cap T_{X_{\iota}}\left(M_{\iota}\right), \iota=1,2$.

Proof. Since $\mathbb{W}_{\iota}, \iota=1,2$ is an inner product space, if $\mathbb{W}_{\iota} \neq \varnothing$, then there exists an orthogonal basis $\left\{\bar{e}_{1}, \cdots, \bar{e}_{i}\right\}$ of $\mathbb{W}_{1}$, an orthogonal basis $\left\{\overline{\bar{e}}_{1}, \cdots, \overline{\bar{e}}_{j}\right\}$ of $\mathbb{W}_{2}$, and an orthogonal complement $\mathbb{W}_{3}$ of $\mathbb{W}_{1} \oplus \mathbb{W}_{2}$ in $\mathbb{W}$, such that $\mathbb{W}=\mathscr{W}_{1} \oplus \mathbb{W}_{2} \oplus \mathbb{W}_{3}$. Denote the orthogonal projections by $P_{i}$ : $T_{\left(x_{1}, x_{2}\right)}\left(M_{1} \times M_{2}\right) \rightarrow T_{x_{\iota}}\left(M_{\iota}\right), \iota=1,2$. Then $P_{2}$ induces a symmetric bilinear form $B$ on $\mathbb{W}_{3}$ (resp. on $\mathbb{W}$ ) given by

$$
B(X, Y)=\left\langle X, P_{2}(Y)\right\rangle,
$$

for every $X, Y \in \mathbb{W}_{3}$ (resp. every $X, Y \in \mathbb{W}$ ). It follows from a diagonalization theorem in linear algebra that there exists an orthonormal basis $\left\{e_{1}, \cdots, e_{k}\right\}$ of $\mathbb{W}_{3}$ (if $\mathbb{W}_{3} \neq \varnothing$ ) such that

$$
\left\langle e_{\ell}, P_{2}\left(e_{\ell^{\prime}}\right)\right\rangle=\delta_{\ell, \ell^{\prime}} \alpha_{\ell}
$$

for some $\alpha_{\ell} \in \mathbb{R}$. Let $P_{1}\left(e_{\ell}\right)=a_{\ell}$, and $P_{2}\left(e_{\ell}\right)=b_{\ell}$. In view of (2)

$$
\left\langle b_{\ell}, b_{\ell^{\prime}}\right\rangle=\left\langle P_{2}\left(e_{\ell}\right), b_{\ell^{\prime}}\right\rangle=\left\langle e_{\ell}, P_{2}\left(e_{\ell^{\prime}}\right)\right\rangle=0,
$$

for every $\ell \neq \ell^{\prime}$, and

$$
\left\langle b_{\ell}, \overline{\bar{e}}_{t}\right\rangle=\left\langle P_{2}\left(e_{\ell}\right), \overline{\bar{e}}_{t}\right\rangle=\left\langle e_{\ell}, P_{2}\left(\overline{\bar{e}}_{t}\right)\right\rangle=\left\langle e_{\ell}, \overline{\bar{e}}_{t}\right\rangle=0 .
$$

It follows that

$$
\left\langle a_{\ell}, a_{\ell^{\prime}}\right\rangle=\left\langle e_{\ell}-b_{\ell}, e_{\ell^{\prime}}-b_{\ell^{\prime}}\right\rangle=\delta_{\ell, \ell^{\prime}}-\left\langle b_{\ell}, b_{\ell^{\prime}}\right\rangle=0,
$$

for every $\ell \neq \ell^{\prime}$. Similarly, $\left\langle a_{\ell}, \bar{e}_{s}\right\rangle=\left\langle e_{\ell}, \bar{e}_{s}\right\rangle=0$. 


\section{Derivation of a trace formula}

Let $S^{n} \times S^{m}$ be the product of Euclidean sphere with metric tensor $\langle$,$\rangle and Riemannian$ connection $\nabla$. We define

$$
\mathcal{V}=\left\{\operatorname{grad}\left(\left.f\right|_{S^{n}}\right): f \in\left(\mathbb{R}^{n+1}\right)^{\prime}\right\} \oplus\left\{\operatorname{grad}\left(\left.g\right|_{S^{m}}\right): g \in\left(\mathbb{R}^{m+1}\right)^{\prime}\right\} .
$$

, where $\left(\mathbb{R}^{n+1}\right)^{\prime}$ is the dual space of $\mathbb{R}^{n+1}$. There is a canonical isomorphism

$$
\mathbb{R}^{n+1} \times \mathbb{R}^{m+1} \rightarrow \mathcal{V}
$$

which associates to $(\nu, w) \in \mathbb{R}^{n+1} \times \mathbb{R}^{m+1}$, the gradient of the function defined by the Euclidean inner product

$$
\left\langle(\nu, w),\left(x_{1}, x_{2}\right)\right\rangle_{\mathbb{R}^{n+1} \times \mathbb{R}^{m+1}}=f_{\nu}\left(x_{1}\right)+g_{w}\left(x_{2}\right)
$$

(where $\left.\left(\mathrm{f}_{\mathrm{v}}\left(\mathrm{x}_{1}\right), \mathrm{g}_{\mathrm{w}}\left(\mathrm{x}_{2}\right)\right)=\left(\left\langle\mathrm{v}, \mathrm{x}_{1}\right\rangle,\left\langle\mathrm{w}, \mathrm{x}_{2}\right\rangle\right)\right)$, i.e., the isomorphism is given by

$$
(\nu, w) \mapsto\left(V\left(x_{1}\right), W\left(x_{2}\right)\right)=\left(\nu-\left\langle v, x_{1}\right\rangle x_{1}, w-\left\langle w, x_{2}\right\rangle x_{2}\right)
$$

for every point $\left(x_{1}, x_{2}\right) \in S^{n} \times S^{m} \subset \mathbb{R}^{n+1} \times \mathbb{R}^{m+1}$. This identification introduces a natural inner product on $\mathcal{V}$ and $\mathcal{V}$ is an $(n+m+2)$-dimensional vector space. For any simple $p$-vector $\xi \in \wedge^{p} T_{x}\left(S^{n} \times S^{m}\right)$ at $x \in S^{n} \times S^{m}$, we associate a quadratic form $Q_{\xi}$ on $\mathcal{V}$ as follows: For $V \in \mathcal{V}$, let $\phi_{t}^{V}$ be the flow generated by $V$,

$$
Q_{\xi}(V)=\left.\frac{d^{2}}{d t^{2}}\left\|\phi_{t^{*}}^{V} \xi\right\|\right|_{t=0} \quad \text { where }\left\|\phi_{t^{*}}^{V} \xi\right\|=\left\langle\phi_{t^{*}}^{V} \xi, \phi_{t^{*}}^{V} \xi\right\rangle^{\frac{1}{2}} .
$$

Likewise, given a $p$-varifold $\mathfrak{S}$ in $S^{n} \times S^{m}$ we can define a quadratic form $Q_{\mathfrak{S}}$ on $\mathcal{V}$

$$
Q_{\mathfrak{S}}(V)=\left.\frac{d^{2}}{d t^{2}} \mathbf{M}\left(\phi_{t^{*}}^{v} \mathfrak{S}\right)\right|_{t=0}
$$

Their traces are linked by

$$
\operatorname{tr} Q_{\mathfrak{S}}=\int_{G_{p}^{\prime}\left(S^{n} \times S^{m}\right)} \operatorname{tr} Q_{\xi} d \mathfrak{S}(\xi),
$$

where $G_{p}^{\prime}\left(S^{n} \times S^{m}\right)$ is the Grassmannian of unoriented $p$-dimensional subspace of $T_{\left(x_{1}, x_{2}\right)}\left(S^{n} \times\right.$ $\left.S^{m}\right)$. In general, to express the second variation formula in terms of geometry of submanifold in $N$, we introduce two tensor fields $\mathscr{A}^{V}$ and $\nabla_{V, \bullet} V$ associated to a vector field $V$ on $N$ as follows:

(1) Given any point $x \in N$ we define a linear map $\mathscr{A}^{V}: T_{x}(N) \rightarrow T_{x}(N)$ by $\mathscr{A}^{V}(X)=\nabla_{X} V$. The map extends uniquely as a derivation to $\wedge^{p} T_{x}(N)$.

(2) At any point $x \in N$, we define also a linear map $\nabla_{V, \bullet} V: T_{x}(N) \rightarrow T_{x}(N)$ by $\nabla_{V, X} V=$ $\nabla_{V} \nabla_{\tilde{X}} V-\nabla_{\nabla_{V} \tilde{X}} V$ where $\tilde{X}$ is any extension of $X$ to a local vector field. Let $\xi=e_{1} \wedge \cdots \wedge e_{p}$ 
where $\left\{e_{1}, \ldots, e_{p}, n_{1}, \ldots, n_{q}\right\}$ is an orthonormal basis for $T_{x}(N)$. It was computed in [5] that for a gradient vector field $V$

$$
\begin{aligned}
\left.\frac{d^{2}}{d t^{2}} \mathbf{M}\left(\phi_{t^{*}}^{V} \mathscr{S}\right)\right|_{t=0} & =\int_{N} Q_{\xi}(V) d\|\mathscr{S}\| \\
& =\int_{N}\left(\sum_{\alpha=1}^{p}\left\langle\mathscr{A}^{V}\left(e_{\alpha}\right), e_{\alpha}\right\rangle\right)^{2}+2 \sum_{\alpha=1}^{p} \sum_{\beta=1}^{q}\left\langle\mathscr{A}^{V}\left(e_{\alpha}\right), n_{\beta}\right\rangle^{2}+\sum_{\alpha=1}^{p}\left\langle\nabla_{V, e_{\alpha}} V, e_{\alpha}\right\rangle d\|\mathscr{S}\| .
\end{aligned}
$$

From now on let a simple $p$-vector $\xi \in \wedge^{p}\left(T_{x}\left(S^{n} \times S^{m}\right)\right)$ at a point $x=\left(x_{1}, x_{2}\right) \in S^{n} \times S^{m}$ be given by $\xi=\bar{e}_{1} \wedge \cdots \wedge \bar{e}_{i} \wedge \overline{\bar{e}}_{1} \wedge \cdots \wedge \overline{\bar{e}}_{j} \wedge e_{1} \wedge \cdots \wedge e_{k}$ where $\bar{e}_{s}, \overline{\bar{e}}_{t}, e_{\ell}$ are as in Theorem 1, with $e_{\ell}=a_{\ell}+b_{\ell}, \bar{e}_{s}, a_{\ell} \in T_{x_{1}}\left(S^{n}\right), \overline{\bar{e}}_{t}, b_{\ell} \in T_{x_{2}}\left(S^{m}\right)$. Note $i, j, k \geq 0$ are such that $i+j+k=p$. That is, throughout this paper, we assume as in Theorem 1 (in which $M_{1}=S^{n}$ and $M_{2}=S^{m}$ ) that

among local frame of $p$ tangent vectors a.e. to a given current in $S^{n} \times S^{m}$, there are $i$ vectors $\bar{e}_{s}$ lying in the first factor of the tangent space (to $S^{n} \times S^{m}$ ), $j$ vectors $\overline{\bar{e}}_{t}$ in the second factor and $k$ vectors $e_{\ell}=a_{\ell}+b_{\ell}$ in both factors.

\section{Proposition 2.}

$\operatorname{tr} Q_{\xi}=i^{2}+(j+k)^{2}-n i-m(j+k)+(2 i-2 j-2 k+4-n+m) \sum_{\ell=1}^{k}\left|a_{\ell}\right|^{2}+2\left(\sum_{\ell=1}^{k}\left|a_{\ell}\right|^{2}\right)^{2}-4 \sum_{\ell=1}^{k}\left|a_{\ell}\right|^{4}$.

Proof. To compute the trace over $\mathcal{V}$, we first consider the gradient vector field $V$ over $S^{n}$ corresponding to a fixed $v \in \mathbb{R}^{n+1}$ (with the Riemannian connection $\bar{\nabla}$ ) through the canonical isomorphism,

$$
\mathscr{A}^{V}\left(e_{\ell}\right)=\nabla_{e_{\ell}} V=\nabla_{a_{\ell}} V=\left(\bar{\nabla}_{a_{\ell}} v-\left\langle\nu, x_{1}^{\prime}\right\rangle x_{1}^{\prime}\right)_{x^{\prime}=x}^{T}=-\left\langle\nu, x_{1}\right\rangle a_{\ell}=-f_{\nu} a_{\ell}
$$

where $\ell=1, \cdots k, x_{1}^{\prime}$ is the projection of $x^{\prime}$ to the first $n+1$ coordinates, and ()$^{T}$ is an orthogonal projection $T_{x_{1}}\left(\mathbb{R}^{n+1}\right) \rightarrow T_{x_{1}}\left(S^{n}\right)$. Similarly, we have

$$
\begin{aligned}
& \mathscr{A}^{V}\left(\bar{e}_{s}\right)=-f_{v} \bar{e}_{s}, \quad \text { where } \quad s=1, \cdots, i \\
& \mathscr{A}^{V}\left(\overline{\bar{e}}_{t}\right)=0 \quad \text { where } t=1, \cdots, j .
\end{aligned}
$$

Furthermore,

$$
\begin{aligned}
& \nabla_{V, e_{\ell}} V=\nabla_{V} \nabla_{e_{\ell}} V=\nabla_{V}\left(-f_{\nu} a_{\ell}\right)=-|V|^{2} a_{\ell} \\
& \nabla_{V, \bar{e}_{s}} V=\nabla_{V}\left(-f_{\nu} \bar{e}_{s}\right)=-|V|^{2} \bar{e}_{s} \\
& \nabla_{V, \overline{\bar{e}}_{t}} V=0 .
\end{aligned}
$$

Therefore,

$$
Q_{\xi}(V)=\left(\sum_{s=1}^{i}\left\langle-f_{\nu} \bar{e}_{s}, \bar{e}_{s}\right\rangle+\sum_{\ell=1}^{k}\left\langle-f_{\nu} a_{\ell}, a_{\ell}\right\rangle\right)^{2}+2 \sum_{s=1}^{i} \sum_{\beta=1}^{q}\left\langle-f_{\nu} \bar{e}_{s}, n_{\beta}\right\rangle^{2}
$$




$$
+2 \sum_{\ell=1}^{k} \sum_{\beta=1}^{q}\left\langle-f_{\nu} a_{\ell}, n_{\beta}\right\rangle^{2}+\sum_{s=1}^{i}-|V|^{2}\left\langle\bar{e}_{s}, \bar{e}_{s}\right\rangle^{2}+\sum_{\ell=1}^{k}-|V|^{2}\left\langle a_{\ell}, a_{\ell}\right\rangle .
$$

Likewise, for $w \in T_{x_{2}}\left(S^{m}\right)$, through the canonical isomorphism $w \longmapsto W$,

$$
\begin{aligned}
Q_{\xi}(W)= & \left(\sum_{t=1}^{j}\left\langle-g_{w} \overline{\bar{e}}_{t}, \overline{\bar{e}}_{t}\right\rangle+\sum_{\ell=1}^{k}\left\langle-g_{w} b_{\ell}, b_{\ell}\right\rangle\right)^{2}+2 \sum_{t=1}^{j} \sum_{\beta=1}^{q}\left\langle-g_{w} \overline{\bar{e}}_{t}, n_{\beta}\right\rangle^{2} \\
& +2 \sum_{\ell=1}^{k} \sum_{\beta=1}^{q}\left\langle-g_{w} b_{\ell}, n_{\beta}\right\rangle^{2}+\sum_{t=1}^{j}-|W|^{2}\left\langle\overline{\bar{e}}_{t}, \overline{\bar{e}}_{t}\right\rangle^{2}+\sum_{\ell=1}^{k}-|W|^{2}\left\langle b_{\ell}, b_{\ell}\right\rangle .
\end{aligned}
$$

Let $\left\{\bar{e}_{1}, \ldots, \bar{e}_{n}\right\}$ and $\left\{\overline{\bar{e}}_{1}, \ldots, \overline{\bar{e}}_{m}\right\}$ be orthonormal bases for $T_{x_{1}}\left(S^{n}\right)$ and $T_{x_{2}}\left(S^{m}\right)$ respectively. We choose orthonormal basis $\left\{x_{1}, x_{2}, \bar{e}_{1}, \ldots, \bar{e}_{n}, \overline{\bar{e}}_{1}, \ldots, \overline{\bar{e}}_{m}\right\}$ for $\mathbb{R}^{n+m+2}$. Through the identification, this fixes an orthonormal basis

$$
\left\{X_{1}, X_{2}, \bar{E}_{1}, \ldots, \bar{E}_{n}, \overline{\bar{E}}_{1}, \cdots, \overline{\bar{E}}_{m}\right\} \text { for } \mathcal{V} .
$$

Let $\mathfrak{B}_{1}=\left\{X_{1}, \bar{E}_{1}, \ldots, \bar{E}_{n}\right\}, \mathfrak{B}_{2}=\left\{X_{2}, \overline{\bar{E}}_{1}, \cdots, \overline{\bar{E}}_{m}\right\}$, and $f_{x_{1}}, g_{x_{2}}, f_{\bar{e}_{1}}, \ldots, f_{\bar{e}_{n}}, g_{\overline{\bar{e}}_{1}}, g_{\overline{\bar{e}}_{m}}$ be the linear functions corresponding to $x_{1}, x_{2}, \bar{e}_{1}, \ldots, \bar{e}_{n}, \overline{\bar{e}}_{1}, \cdots \overline{\bar{e}}_{m}$. Then obviously, $X_{1}(x)=X_{2}(x)=f_{\bar{e}_{1}}(x)=$ $\cdots=f_{\bar{e}_{n}}(x)=g_{\overline{\bar{e}}_{1}}(x)=\cdots=g_{\overline{\bar{e}}_{m}}(x)=0$ and $\left|\bar{E}_{1}(x)\right|=\cdots=\left|\bar{E}_{n}(x)\right|=\left|\overline{\bar{E}}_{1}(x)\right|=\cdots=\left|\overline{\bar{E}}_{m}(x)\right|=$ $\left|f_{x_{1}}\left(x_{1}\right)\right|=\left|g_{x_{2}}\left(x_{2}\right)\right|=1$.

Therefore, the trace tr over $\mathcal{V}$

$$
\begin{aligned}
\operatorname{tr}=\operatorname{tr} Q_{\xi}= & \sum_{V \in \mathfrak{B}_{1}} Q_{\xi}(V)+\sum_{W \in \mathfrak{B}_{2}} Q_{\xi}(W) \\
= & \left(i+\sum_{\ell=1}^{k}\left|a_{\ell}\right|^{2}\right)^{2}+\left(j+\sum_{\ell=1}^{k}\left|b_{\ell}\right|^{2}\right)^{2} \\
& +2\left(\sum_{\ell=1}^{k} \sum_{\beta=1}^{q}\left\langle a_{\ell}, n_{\beta}\right\rangle^{2}+\sum_{\ell=1}^{k} \sum_{\beta=1}^{q}\left\langle b_{\ell}, n_{\beta}\right\rangle^{2}\right) \\
& -n\left(i+\sum_{\ell=1}^{k}\left|a_{\ell}\right|^{2}\right)-m\left(j+\sum_{\ell=1}^{k}\left|b_{\ell}\right|^{2}\right) .
\end{aligned}
$$

It follows from Theorem 1 that for $1 \leq \ell \leq k$,

$$
\begin{aligned}
a_{\ell} & =\sum_{s=1}^{i}\left\langle a_{\ell}, \bar{e}_{s}\right\rangle \bar{e}_{s}+\sum_{t=1}^{j}\left\langle a_{\ell}, \overline{\bar{e}}_{t}\right\rangle \overline{\bar{e}}_{t}+\sum_{\beta=1}^{q}\left\langle a_{\ell}, n_{\beta}\right\rangle n_{\beta}+\sum_{h=1}^{k}\left\langle a_{\ell}, a_{h}+b_{h}\right\rangle\left(a_{h}+b_{h}\right) \\
& =\sum_{\beta=1}^{q}\left\langle a_{\ell}, n_{\beta}\right\rangle n_{\beta}+\left|a_{\ell}\right|^{2}\left(a_{\ell}+b_{\ell}\right) .
\end{aligned}
$$

Hence

$$
\left|a_{\ell}\right|^{2}=\sum_{\beta=1}^{q}\left\langle a_{\ell}, n_{\beta}\right\rangle^{2}+\left|a_{\ell}\right|^{4}
$$


Therefore,

$$
\sum_{\beta=1}^{q}\left\langle a_{\ell}, n_{\beta}\right\rangle^{2}=\left|a_{\ell}\right|^{2}-\left|a_{\ell}\right|^{4} .
$$

Likewise,

$$
\sum_{\beta=1}^{q}\left\langle b_{\ell}, n_{\beta}\right\rangle^{2}=\left|b_{\ell}\right|^{2}-\left|b_{\ell}\right|^{4}
$$

Consequently, it follows from (3) and (4) that

$$
\begin{aligned}
\operatorname{tr}=\operatorname{tr} Q_{\xi}= & i^{2}+2 i \sum_{\ell=1}^{k}\left|a_{\ell}\right|^{2}+\left(\sum_{\ell=1}^{k}\left|a_{\ell}\right|^{2}\right)^{2}+j^{2}+2 j\left(\sum_{\ell=1}^{k}\left|b_{\ell}\right|^{2}\right) \\
& +\left(\sum_{\ell=1}^{k}\left|b_{\ell}\right|^{2}\right)^{2}+4 \sum_{\ell=1}^{k}\left|a_{\ell}\right|^{2}\left|b_{\ell}\right|^{2}-n\left(i+\sum_{\ell=1}^{k}\left|a_{\ell}\right|^{2}\right)-m\left(j+\sum_{\ell=1}^{k}\left|b_{\ell}\right|^{2}\right) \\
= & i^{2}+(j+k)^{2}-n i-m(j+k)+(2 i-2 j-2 k+4-n+m) \sum_{\ell=1}^{k}\left|a_{\ell}\right|^{2} \\
& +2\left(\sum_{\ell=1}^{k}\left|a_{\ell}\right|^{2}\right)^{2}-4 \sum_{\ell=1}^{k}\left|a_{\ell}\right|^{4} .
\end{aligned}
$$

When we consider the case in a single sphere $S^{n}$ where $m=j=k=\left|a_{k}\right|=0$, the proposition yields $\operatorname{tr}=i(i-n)<0$ and

Theorem 3 (Lawson and Simons [7]). There are no closed stable varifolds in $S^{n}$, in particular there are no closed stable rectifiable currents for any finitely generated abelian group $G$.

\section{Proof of the classification theorem}

The trace estimates vary as the relative size of the degree $p$ of an integral current to the dimensions of $S^{n}$ and $S^{m}$ varies. We divide them into five major cases.

Case 1. $p \leq m<n \quad(i=j=0, k=p)$

(i) $1<p$

Since $2\left|a_{h}\right|^{2}\left|a_{\ell}\right|^{2} \leq\left|a_{h}\right|^{4}+\left|a_{\ell}\right|^{4}$ and $\left|a_{\ell}\right|^{4} \leq\left|a_{\ell}\right|^{2} \leq 1$,

$$
\begin{aligned}
\operatorname{tr} & =p^{2}-m p+(-2 p+4-n+m)\left(\sum_{\ell=1}^{k}\left|a_{\ell}\right|^{2}\right)+4 \sum_{h \neq \ell}\left|a_{\ell}\right|^{2}\left|a_{h}\right|^{2}-2 \sum_{\ell=1}^{k}\left|a_{\ell}\right|^{4} \\
& \leq p^{2}-m p+(-2 p+4-n+m)\left(\sum_{\ell=1}^{k}\left|a_{\ell}\right|^{2}\right)+2(p-1) \sum_{\ell=1}^{k}\left|a_{\ell}\right|^{4}-2 \sum_{\ell=1}^{k}\left|a_{\ell}\right|^{4} \\
& \leq p(p-m)-(n-m) \sum_{\ell=1}^{k}\left|a_{\ell}\right|^{2}
\end{aligned}
$$




$$
\leq 0 \text { and " }=\text { " holds if and only if } p=m \text { and } a_{\ell}=0 \text {. }
$$

(ii) $1=p<m<n$

$$
\begin{aligned}
\operatorname{tr} & =1-m+(2-n+m)\left|a_{1}\right|^{2}-2\left|a_{1}\right|^{4} \\
& \leq 1-m+\left|a_{1}\right|^{2}-2\left|a_{1}\right|^{4}<0 .
\end{aligned}
$$

(iii) $1=p=m<2 \leq n$

$$
\begin{aligned}
\operatorname{tr} & =(3-n)\left|a_{1}\right|^{2}-2\left|a_{1}\right|^{4} \\
& \leq(1-n)\left|a_{1}\right|^{4} \\
& \leq 0 \quad \text { and " }=\text { " holds if and only if } a_{1}=0 .
\end{aligned}
$$

Case 2. $\quad m<n \leq p \quad(i=p-m, j=p-n, k=-p+m+n)$

(i) $k>1$

$$
\begin{aligned}
\operatorname{tr} & =i^{2}+(j+k)^{2}-n i-m(j+k)+(2 i-2 j-2 k+4-n+m) \sum_{\ell=1}^{k}\left|a_{\ell}\right|^{2} \\
& +4 \sum_{h \neq \ell}\left|a_{\ell}\right|^{2}\left|a_{h}\right|^{2}-2 \sum_{\ell=1}^{k}\left|a_{\ell}\right|^{4} \\
\leq & (p-m-n)(p-m)+(2 i-2 j-n+m) \sum_{\ell=1}^{k}\left|a_{\ell}\right|^{2} \\
\leq & (p-m-n)(p-m)+(n-m) \sum_{\ell=1}^{k}\left|a_{\ell}\right|^{2} \\
\leq & (p-m-n)(p-n)+(m-n) \sum_{\ell=1}^{k}\left|b_{\ell}\right|^{2} \\
\leq & 0 \quad \text { and } "=" \text { holds if and only if } p=n, b_{\ell}=0 .
\end{aligned}
$$

(ii) $k=1, \quad 1<m<n \leq p$

$$
\begin{aligned}
\operatorname{tr} & =-(p-m)+(n-m+2)\left|a_{1}\right|^{2}-2\left|a_{1}\right|^{4} \\
& =(1-n)+(n-m)+2\left|a_{1}\right|^{2}-2\left|a_{1}\right|^{4} \\
& \leq-1+\frac{\sin ^{2} 2 \theta}{2} \quad \text { where } \cos ^{2} \theta=\left|a_{1}\right|^{2} \\
& <0 .
\end{aligned}
$$

(iii) $k=1, \quad 1=m<2 \leq n \leq p$

$$
\begin{aligned}
\operatorname{tr} & =(1-n)+(n+1)\left|a_{1}\right|^{2}-2\left|a_{1}\right|^{4} \\
& =-\left(\left|a_{1}\right|^{2}-1\right)\left(2\left|a_{1}\right|^{2}-(n+1)\right)
\end{aligned}
$$




$$
\leq 0 \quad \text { and } \quad \text { "=" holds if and only if }\left|a_{1}\right|=1
$$

Case 3. $m \leq p \leq n \quad(i=p-m, j=0, k=m)$

(i) $k>2, \quad 2 p-m-n \leq 0$

$$
\begin{aligned}
\operatorname{tr} & =(p-m)(p-m-n)+(2 p-3 m-n+4) \sum_{\ell=1}^{k}\left|a_{\ell}\right|^{2}+2\left(\sum_{\ell=1}^{k}\left|a_{\ell}\right|^{2}\right)^{2}-4 \sum_{\ell=1}^{k}\left|a_{\ell}\right|^{4} \\
& \leq(p-m)(p-m-n)+(2 p-m-n) \sum_{\ell=1}^{k}\left|a_{\ell}\right|^{2} \\
& \leq 0 \quad \text { and " }=" \text { holds if and only if } p=m<n, a_{\ell}=0 \text { or } p=m=n,\left|a_{\ell}\right|=0 \text { or } 1 .
\end{aligned}
$$

(ii) $k>2, \quad 2 p-m-n>0$

$$
\begin{aligned}
\operatorname{tr} & \leq(p-m)(p-m-n)+(p-m-n) m+p m \\
& =(p-n) p \\
& \leq 0 \quad \text { and } \quad "=\text { " holds if and only if } m<p=n \text { and }\left|a_{\ell}\right|=1 .
\end{aligned}
$$

(iii) $k=2, \quad 2 p-m-n \leq 0$

$$
\begin{aligned}
\operatorname{tr} & =(p-m)(p-m-n)+(2 p-m-n) \sum_{\ell=1}^{k}\left|a_{\ell}\right|^{2} \\
& \leq 0 \quad \text { and } "=" \text { holds if and only if } p=m<n, a_{\ell}=0 \text { or } p=m=n=2,\left|a_{1}\right|=\left|a_{2}\right| .
\end{aligned}
$$

(iv) $k=2, \quad 2 p-m-n>0$

$$
\begin{aligned}
\operatorname{tr} & \leq(p-m)(p-m-n)+(2 p-m-n) k \\
& =(p-n) p \\
& \leq 0 \quad \text { and } \quad "=\text { " holds if and only if } m<p=n \text { and }\left|a_{\ell}\right|=1 .
\end{aligned}
$$

(v) $k=1, m \leq p \leq n$ and $m<n$

See Case 2(iii).

Case 4. $2<m=n$

(i) $\quad k>1, \quad 2<m=n \leq p \quad(i=p-m, j=p-m, k=2 m-p)$

$$
\begin{aligned}
\operatorname{tr}= & i^{2}+(j+k)^{2}-n i-m(j+k)+(2 i-2 j-2 k+4-n+m) \sum_{\ell=1}^{k}\left|a_{\ell}\right|^{2} \\
& +4 \sum_{\ell^{\prime} \neq \ell}\left|a_{\ell}\right|^{2}\left|a_{\ell^{\prime}}\right|^{2}-2 \sum_{\ell=1}^{k}\left|a_{\ell}\right|^{4}
\end{aligned}
$$




$$
\begin{aligned}
& \leq(p-2 m)(p-m)+(2 p-4 m+4) \sum_{\ell=1}^{k}\left|a_{\ell}\right|^{2}+(4 m-2 p-4)\left|a_{\ell}\right|^{4} \\
& \leq 0 \quad \text { and } \quad "=" \text { holds if and only if }\left|a_{\ell}\right|=1 \text { or } 0, \text { and } p=m .
\end{aligned}
$$

(ii) $k=1, \quad 2<m=n<p$

$$
\begin{aligned}
\operatorname{tr} & =-(p-m)+2\left|a_{1}\right|^{2}-2\left|a_{1}\right|^{4} \\
& =-(m-1)+2\left|a_{1}\right|^{2}-2\left|a_{1}\right|^{4} \\
& <0 .
\end{aligned}
$$

(iii) $\quad 3 \leq m=n$ and $\quad p \leq m, \quad k>1 \quad(i=j=0, k=p)$

$$
\begin{aligned}
\operatorname{tr} & =p^{2}-m p+(-2 p+4) \sum_{\ell=1}^{k}\left|a_{\ell}\right|^{2}+2\left(\sum_{\ell=1}^{k}\left|a_{\ell}\right|^{2}\right)^{2}-4 \sum_{\ell=}^{k}\left|a_{\ell}\right|^{4} \\
& \leq p(p-m) \\
& \leq 0 \quad \text { and } "=\text { " holds if and only if }\left|a_{\ell}\right|=0 \text { or } 1, p=m .
\end{aligned}
$$

(iv) $\quad 2<m=n$ and $p \leq m, k=1$

$$
\begin{aligned}
\operatorname{tr} & =1-m+2\left|a_{1}\right|^{2}-2\left|a_{1}\right|^{4} \\
& <0 .
\end{aligned}
$$

Case 5. $n=m=2$

(i) $\quad p=1 \quad(i=j=0, k=1)$

$$
\begin{aligned}
\operatorname{tr} & =-\left(1-\left|a_{1}\right|^{2}\right)^{2}-\left|a_{1}\right|^{4} \\
& <0 .
\end{aligned}
$$

(ii) $p=2,(i=j=0, k=2)$

Let

$$
\begin{aligned}
e_{1} & =\cos \theta_{1} \bar{e}_{1}+\sin \theta_{1} \overline{\bar{e}}_{1} \\
e_{2} & =\cos \theta_{2} \bar{e}_{2}+\sin \theta_{2} \overline{\bar{e}}_{2} \\
\operatorname{tr} & =4\left|a_{1}\right|^{2}\left|a_{2}\right|^{2}-2\left|a_{1}\right|^{4}-2\left|a_{2}\right|^{4} \\
& =-2\left(\left|a_{1}\right|^{2}-\left|a_{2}\right|^{2}\right)^{2} \\
& \leq 0 \quad \text { and } "=" \text { holds } \\
& \quad \text { if and only if }\left|a_{1}\right|=\left|a_{2}\right|, \\
& \quad \text { if and only if }\left|\cos \theta_{1}\right|=\left|\cos \theta_{2}\right| \text { and }\left|\sin \theta_{1}\right|=\left|\sin \theta_{2}\right|,
\end{aligned}
$$




$$
\text { if and only if } J\left(e_{1} \wedge e_{2}\right)=0 \text { or } J\left(e_{1} \wedge e_{2}\right) \perp\left(e_{1} \wedge e_{2}\right) \text {. }
$$

We note that if $\operatorname{tr}<0$, then there exists a vector field $V \in \mathfrak{B}_{1}$, or $W \in \mathfrak{B}_{2}$, along which the variation decreases the mass of $T$. Hence $T$ is not stable.

By the result of Case 5, the only stable currents in $S^{2} \times S^{2}$ are holomorphic or anti-holomorphic or sums of these. The estimates in Cases 1 through 4 indicate that if $T$ is a $p$-dimensional stable current in $S^{n} \times S^{m}$ where $p$ and $q \neq 1$ or 2 , then

$$
p=n \quad \text { and } \quad\left|b_{\ell}\right|=0
$$

or

$$
p=m \quad \text { and } \quad\left|a_{\ell}\right|=0 .
$$

Without loss of generality, let $T$ be a current such that $T_{x}=\overline{\bar{e}}_{1} \wedge \cdots \wedge \overline{\bar{e}}_{m},\|T\|$ a.e. Take a dual version of current $T$

$$
T=u d x^{1} \wedge \cdots \wedge d x^{n}
$$

where a distribution coefficient $u \in \mathscr{E}_{0}\left(S^{n} \times S^{m}\right)$ is given by

$$
u(f)=T\left(f \overline{\bar{e}}_{1} \wedge \cdots \wedge \overline{\bar{e}}_{m}\right) \text { for } f \in \mathscr{E}^{0}\left(S^{n} \times S^{m}\right) .
$$

Since

$$
\begin{aligned}
0 & =\partial T=\frac{\partial u}{\partial y_{j}} d y^{j} \wedge d x^{1} \wedge \cdots \wedge d x^{n}, \\
\frac{\partial u}{\partial y_{j}} & \equiv 0 \quad \text { for } \quad j=1, \ldots m .
\end{aligned}
$$

Define

$$
\phi_{t}^{j}(x, y)=\left(x, y_{1}, \ldots, y_{j}+t, \ldots, y_{m}\right) \quad \text { for } \quad j=1, \ldots, m
$$

Then

$$
\phi_{t^{*}}^{j} T \quad \text { for } \quad j=1, \ldots, m .
$$

It follows that $T$ and therefore supp $T$ are invariant under all translations in the $y$-direction. Hence support of $T$ is a union of $y$-planes. Since $T$ is a closed stationary rectifiable current, the Hausdorff $n$-measure of $\operatorname{supp}(T)$ is finite [3]. It follows that $\operatorname{supp} T$ is a finite union of $y$ planes. By [4] any closed rectifiable $m$-current supported in an $m$-dimensional submanifold is given, up to integral multiples, by integration over that submanifold.

Corollary 1. For $p \neq n$ or $m$, there are no closed stable integral $p$-currents on $S^{n} \times S^{m}$. In fact there are no closed stable rectifiable $p$-currents in $R_{p}\left(S^{n} \times S^{m}, G\right)$ for any finitely generated abelian group $G$ and there are no closed stable $p$-dimensional varifolds in $S^{n} \times S^{m}$.

Proof. Since the proof involves only a point by point computation, and since the result is independent of orientation, a similar formula holds for varifolds. 


\section{References}

[1] M. Ara, Instability and nonexistence theorems for F-harmonic maps, Illinois J. Math. 45 (2001), 657-679.

[2] E. Bombieri, E. de Giorgi and E. Giusti, Minimal cones and the Bernstein problem, Invent. Math., 7(1969), 243-268.

[3] H. Federer, Geometric Measure Theory, Berlin-Heidelberg-New York: Springer (1969).

[4] H. Federer and W. H. Fleming, Normal and integral currents, Ann. of Math., 72 (1960), 458-520.

[5] R. Howard and S. W. Wei, Non-existence of stable harmonic maps to and from certain homogeneous spaces and submanifolds of Euclidean space Trans. A.M.S. 294 No. 1 (1986), 319-331.

[6] H. B. Lawson Jr, The equivarinnt Plateau problem and interior regularity, Trans. A.M.S., 173 (1972), 231-249.

[7] H. B. Lawson, Jr. and J. Simons, On stable currents and their application to global problems in real and complex geometry, Ann. of Math. (2), 98(1973), 927-950.

[8] H. B. Lawson Jr, The stable homology of a flat torus, Math. Scand., 36(1975), 49-73.

[9] J. Simons, Minimal varieties in riemannian manifolds, Ann. of Math. (2), 88 (1968), 62-105.

[10] J. L. Synge, On the connectivity of spaces of positive curvature, Quart. J. Math. Oxford Ser. (1), (1936), 316-320

[11] S. W. Wei, Plateau's problem. in symmetric spaces, Nonlinear Anal., 12 (1988), 749-760.

[12] S. W. Wei, On topological vanishing theorems and the stability of Yang-Mills fields, Indiana Univ. Math. J., 33 (1984), 511-529.

[13] S.W. Wei, On 1-harmonic functions, SIGMA 3, no. 127, (2007), 1-10.

[14] S. W. Wei, Minimality, stability, and Plateau problem, Thesis, Berkeley, 1980.

[15] S. W. Wei, An extrinsic average variational method, Recent developments in geometry (Los Angeles, CA, 1987), Contemp. Math. vol 101, Amer. Math. Soc., Providence, RI, (1989), 55-78.

[16] S.W. Wei, Representing Homotopy Groups and Spaces of Maps by p-harmonic maps, Indiana University Mathematics Journal, 47(1998), 625-669

[17] S. W. Wei, and L. Wu, Homotopy groups and p-Harmonic Maps, Commun. Math. Anal. Conf., 01 (2008) $40-45$.

[18] S.W. Wei and C.M. Yau, Regularity of p-energy minimizing maps and p-superstrongly unstable indices J. Geom. Analysis 4 (1994), 247-272.

Department of Mathematics, University Of Oklahoma, Norman, Oklahoma 73019- 0315, U.S.A.

E-mail: wwei0ou.edu 